\title{
Autoeficácia na Solução de Problemas Matemáticos e Variáveis Relacionadas
}

\author{
Márcia Regina Ferreira de Brito \\ Faculdade de Educação da Universidade Estadual de Campinas, Campinas, SP, Brasil \\ Liliane Ferreira Neves Inglez de Souza ${ }^{1}$ \\ Instituto de Ciências Humanas da Universidade Paulista, Limeira, SP, Brasil \\ Faculdade de Administração e Artes de Limeira, Limeira, SP, Brasil
}

\begin{abstract}
Resumo
Baseado na Teoria Social Cognitiva de Albert Bandura, o presente estudo teve como objetivo verificar a existência de relações entre as crenças de autoeficácia e o desempenho na solução de problemas matemáticos. Buscou-se também analisar as correlações entre os instrumentos e avaliar o autoconceito matemático e a autoeficácia para a aprendizagem autorregulada, além de outra variáveis pertinentes. Os participantes foram 131 estudantes da quinta série do período matutino, sendo 72 do gênero masculino e 59 do gênero feminino, matriculados em uma escola confessional privada de uma cidade de grande porte da região sudeste do país. A coleta de dados foi conduzida em período normal de aula e incluiu a aplicação dos seguintes instrumentos: escala autoeficácia para aprendizagem autorregulada, escala de autoconceito, escala de autoeficácia matemática, escala de importância da Matemática, e um instrumento de autoeficácia matemática. A análise dos resultados apontou que tanto a autoeficácia matemática, quanto a autoeficácia para autorregulação estavam relacionadas ao desempenho na tarefa de solução de problemas. Estes dados trazem implicações ao processo de ensino-aprendizagem de Matemática que deve compreender, além do desenvolvimento de habilidades, a construção de autopercepções favoráveis do estudante.
\end{abstract}

Palavras-chave: Auto-eficácia, solução de problemas, Educação Matemática.

\section{Self-Efficacy in Mathematics Problem Solving and Related Variables}

\begin{abstract}
Based on Albert Bandura' Social Cognitive Theory the objective of the present study was to verify the existence of relations between self-efficacy beliefs and performance in mathematical problem solving. It also sought to evaluate the mathematical self-concept and self-efficacy for self-regulated learning. We attempted to study the correlation between the instruments and evaluate the mathematical self-concept and self-efficacy for self-regulated learning, and other relevant variables. Participants were 131 fifth graders, 72 males and 59 females from in a private religious school in a large city in the southeast of the country. Data have been collected in regular class schedules and included the following instruments: self-efficacy scale for regulated learning, self-concept-scale, importance of mathematics, and mathematics self-efficacy instruments 1 and 2. Results revealed that both mathematics self-efficacy, and self-efficacy for self-regulated learning were related to performance on solving problems. These data have
\end{abstract}

Endereço para correspondência: Rua José Dirceu Bais, 296, Residencial Flora, Limeira, SP, Brasil 13482-243.

E-mail:1fneves@terra.com.br 
implications for the process of teaching and learning of mathematics which shall include, in addition to developing abilities, building students' suitable self-perceptions.

Keywords: Self-efficacy, problem-solving, Mathematics Education.

\section{Auto-Eficacia en la Resolución de Problemas Matemáticos y Variables Asociadas}

\section{Resumen}

Fundamentado en la teoría social cognitiva de Albert Bandura, el presente investigó la existencia de relaciones entre las creencias de autoeficacia y el rendimiento en la solución de problemas matemáticos. Hemos tratado de estudiar la correlación entre los instrumentos y evaluar el autoconcepto matemático, la auto-eficacia para el aprendizaje auto-regulado, y otras variables pertinentes. Los participantes fueron 131 estudiantes de quinto grado del período de la mañana, 72 varones y 59 mujeres inscritos en una escuela religiosa privada en una gran ciudad en el sureste del país. La colecta de datos se llevó a cabo en la clase normal y estén incluidos los siguientes instrumentos: escala de autoeficacia para el aprendizaje autorregulado, escala de autoconcepto, escala de la importancia de las matemáticas, y un instrumento de auto-eficacia matemática. Los resultados mostraron que tanto la auto-eficacia matemática y auto-eficacia de la autorregulación se relacionam con el rendimiento en la tarea de resolver los problemas. Estos datos tienen implicaciones para el proceso de enseñanza y aprendizaje de las matemáticas, que incluirá, además de desarrollar habilidades, la construcción de auto-percepción a favor del estudiante.

Palabras clave: Auto-eficacia, solución de problemas, Educación Matemática.

Ao longo de sua vida acadêmica o estudante desenvolve crenças na própria capacidade frente às exigências escolares e isso se transforma, em interação com o ambiente, em uma das principais influências no sucesso ou fracasso escolar. De acordo com Bandura $(1977,1986)$ os indivíduos desenvolvem crenças autorreferenciadas e apresentam comportamentos proativos e autorregulados e esses referenciais ordenam o comportamento e as ações do indivíduo no ambiente através do controle sobre o pensamento, os sentimentos e as ações.

A aprendizagem envolve mudança e ocorre quando o indivíduo se defronta com uma tarefa desconhecida, através da qual ele vai aprender os procedimentos e componentes da tarefa. Isso implica em mudanças cognitivas, afetivas e comportamentais. Posteriormente, ao defrontar-se com a mesma tarefa ou tarefas similares conhecidas, ele colocará em ação os mecanismos desenvolvidos ao longo do tempo e em diferentes situações, escolhendo cursos de ação congruentes com as crenças que possui a respeito de sua capacidade e as exigências da tarefa. Ao se defrontar com uma tarefa, o indivíduo adquire familiaridade com as exigências e características da mesma e, conforme vai se familiarizando com a tarefa, também vai adquirindo maior confiança na realização de atividades similares, desenvolvendo confiança na própria capacidade de executar, com sucesso, uma determinada ação (Pajares \& Schunk, 2001).

Assim, quando o indivíduo se defronta com uma situação desconhecida, ele põe em ação as crenças de autoeficácia na aprendizagem, ao passo que, quando a tarefa já é conhecida, entram em ação as crenças de autoeficácia no desempenho. Os estudos a respeito das habilidades e da afetividade na sala de aula e as relações com aspectos cognitivos e motores ganharam novo impulso em décadas recentes. Pesquisas a respeito da motivação e seus componentes foram decisivas para estabelecer critérios e relações entre construtos e permitiram a construção de instrumentos mais adequados ao estudo desses aspectos. Contribuiu também para isso o refinamento dos métodos estatísticos para tratamento dos dados obtidos e a construção de escalas mais 
sofisticadas. A maioria dos estudos revistos no presente trabalho mostram estreita relação entre construtos latentes como autoconceito, autoeficácia, autorregulação, etc. Dado o entrelaçamento desses conceitos muitas vezes é difícil diferenciá-los e verificar a hierarquia, a origem e procedência de cada um deles.

De acordo com Shavelson, Hubner e Stanton (1976) autoconceito é definido como a percepção de uma pessoa sobre si mesma, sendo que estas percepções são formadas e influenciadas principalmente através de experiências com o ambiente e outras pessoas que são significativas. $\mathrm{O}$ autoconceito e a autoeficácia muitas vezes são confundidos, mas autoeficácia se refere a um contexto específico e está relacionada à percepção de competência do indivíduo para a realização de uma tarefa específica. $\mathrm{O}$ autoconceito é mais geral e envolve, além das percepções do sujeito sobre sua própria competência, as crenças de autoestima que constituem um componente avaliativo do autoconceito. Apesar disso, muitos autores tratam estes conceitos como um mesmo constructo (Pajares \& Schunk, 2001). Esses autores apontaram que o autoconceito e as crenças de autoeficácia representam diferentes visões que o sujeito tem de si mesmo, sendo que a autoeficácia está ligada à crença do sujeito sobre sua capacidade de agir, de realizar alguma atividade. Perguntas como: "Posso solucionar esse problema? Posso responder essa questão da prova?" se referem ao grau de confiança que o indivíduo tem para resolver o problema ou solucionar a questão da prova. Esse grau de confiança está estreitamente vinculado a atingir, com sucesso, o objetivo proposto, ou seja, "poder" ou "conseguir" realizar a tarefa. Já ao autoconceito reflete percepções mais gerais do indivíduo, incluindo os sentimentos do indivíduo. Assim, ao se defrontar com determinado problema, as questões que o sujeito se faz seriam do tipo "Como eu me sinto quando soluciono um problema?"; "Por que fico ansioso na prova de Matemática?" ou ainda: "Sou um bom estudante de Matemática?".

O modelo hierárquico proposto por Shavelson et al. (1976) apresenta o autoconceito como descritivo e avaliativo, multidimensional e com uma estrutura hierarquicamente organizada; é dinâmico e estrutura-se de maneira cada vez mais complexa e diferenciada de acordo com o desenvolvimento do indivíduo. Isso implica em uma percepção dinâmica de si mesmo em áreas específicas (como a Matemática) até áreas mais amplas (acadêmicas e não acadêmicas), sendo que o conjunto dessas percepções refere-se ao autoconceito mais geral.

\section{Autoeficácia}

A teoria social cognitiva proposta por Bandura (1986, 1997) compreende o funcionamento humano em termos de uma vasta potencialidade, considerando que as pessoas têm algumas capacidades que lhes permitem exercer certo controle sobre eventos que afetam suas vidas (Bandura, 1986). Dentre estas, encontra-se a capacidade autorreflexiva que envolve principalmente os pensamentos autorreferenciados, ou as crenças que as pessoas têm a respeito de si mesmas. A autorreflexão permite às pessoas analisarem suas experiências e seus próprios processos de pensamento, proporcionando uma organização das autopercepções.

Inclui-se neste contexto, a autoeficácia, definida por Bandura (1997, p. 3) como uma “crença na própria capacidade de organizar e executar cursos de ações requeridas para produzir determinadas realizações". Segundo Bandura (1986) dentre as crenças autorreferenciadas, nenhuma é mais central do que autoeficácia, visto que esta pode influenciar os processos de motivação, as escolhas que os indivíduos realizam, os cursos de ação que estes tomam e suas reações emocionais, afetando de diversas maneiras essas realizações.

Segundo Bandura $(1986,1997)$ a autoeficácia exerce seu papel no desempenho principalmente através de quatro processos:

Processos Cognitivos: relacionados à previsão ou expectativa acerca das possíveis conseqüências que determinada ação provocará, incluindo os objetivos desejados (Bandura, 1986, 1997, 1995/1999). De acordo com o modelo teórico, pessoas com percepções de autoeficácia mais elevadas geralmente possuem expectativas 
mais altas com relação ao desempenho, mantendo crenças de que obterão sucesso.

Processos Motivacionais: A autoeficácia se relaciona a diversos constructos motivacionais, como expectativas, metas, quantidade de esforço e persistência despendidos nas tarefas (Bandura, 1995/1999). Assim, postula-se que as pessoas investem interesse mais duradouro em atividades nas quais se julgam capazes, e das quais obtém satisfação quando atingem o domínio necessário.

Processos Afetivos: Segundo Bandura (1995/1999), as crenças das pessoas em suas capacidades afetam a quantidade de stress e ansiedade que estas experimentam em situações consideradas difíceis ou ameaçadoras. Em geral, a pesquisa tem demonstrado relações inversas entre autoeficácia e ansiedade.

Processos de Seleção: As percepções de autoeficácia também têm sido relacionadas às escolhas realizadas, visto que em geral, as pessoas costumam engajar-se em atividades que acreditam estar de acordo com as habilidades que julgam possuir.

Desta maneira, a autoeficácia tem sido considerada um importante elemento preditivo do desempenho acadêmico. Por exemplo, o estudo de Soares e Oliveira (2011) avaliou constructos como raciocínio verbal e desempenho escolar, apontando que a autoeficácias se apresentou tão importante quanto as demais variáveis para um bom desempenho escolar.

No entanto, não se pode apontar que a autoeficácia determine o desempenho. Para Bandura $(1986,1997)$ a relação entre as crenças e o desempenho é recíproca, e esta formulação teórica tem encontrado suporte na pesquisa. Um exemplo é o estudo conduzido por Williams e Williams (2010) com adolescentes de 33 países, no qual foram encontradas evidências apoiando as ideias de Bandura, de que existe uma relação recíproca entre autoeficácia e desempenho.

De acordo com Pajares (2005) o conhecimento, pelos professores, a respeito das crenças de autoeficácia é de extrema importância, pois permite a eles atuar de forma a influir nos estados emocionais dos estudantes corrigindo eventuais falhas na percepção da capacidade de reali- zação e nos hábitos de pensamento, melhorar as capacidades acadêmicas e de autorregulação dos estudantes além de criar condições de ensino que permitam levar o estudante ao sucesso.

É importante ressaltar que a autoeficácia se forma a partir de quatro fontes de informação: experiências diretas, ou desempenhos anteriores; experiências vicárias, ou a partir da observação de outros; a persuasão social, vinculada às informações recebidas pelas pessoas acerca de seus desempenhos e capacidades e os estados fisiológicos e afetivos. Desta forma, no contexto escolar, os estudantes lidam com tarefas que conseguem ou não realizar com sucesso, recebem uma quantidade de informações sobre seu desempenho, observam os desempenhos dos pares, e experienciam estados fisiológicos ou afetivos. Estas situações ajudam a moldar as crenças de autoeficácia referentes às disciplinas escolares, confirmando a relevância das experiências do aluno com a Matemática na formação das crenças de autoeficácia referentes a esta disciplina.

\section{Solução de Problemas e Crenças de Autoeficácia}

Por se tratar de um julgamento pessoal de capacidade relativa a um determinado domínio, a autoeficácia não se refere especificamente ao autoconceito geral ou à capacidade de um indivíduo em uma variedade de circunstâncias, mas sim ao que o mesmo acredita ser capaz de realizar em um domínio específico. Esta crença pode variar fortemente de acordo com a tarefa ou domínio, e mesmo no contexto escolar, diferencia-se de acordo com as disciplinas escolares. Alguns estudos fazem referência à autoeficácia acadêmica (Arens, Craven, Yeung, \& Hasselhorn, 2011; Rodrigues \& Barreira, 2007; Soares \& Oliveira, 2011), mas muitos se atêm a aspectos mais específicos considerando, por exemplo, autoeficácia matemática (Chen, 2003; Torisu \& Ferreira, 2011).

Dentre as pesquisas sobre autoeficácia matemática, tem se buscado compreender as relações deste constructo com aspectos cognitivos, afetivos e motivacionais, e alguns aspectos estudados tem sido: desempenho escolar (Chen, 
2003; Dobarro \& Brito, 2010; Hackett \& Betz, 1989; Lent, Brown, \& Larkin, 1984; Pajares, 1996), autorregulação (Inglez de Souza, 2008), metas e orientações motivacionais (Bong, 2004; Stevens, Olivarez, Lan, \& Tallent-Runnels, 2004; Wolters \& Pintrich, 1998; Yailagh, Lloyd, \& Walsh, 2009), ansiedade frente às avaliações através de provas e exames (Bandalos, Yates, \& Thorndike-Christ, 1995), autoconceito (Inglez de Souza \& Brito, 2008; Shiomi, 1992), atribuições de causalidade para sucesso e fracasso nesta disciplina (Barros, 1996; S. P. Neves \& Faria, 2007) atitudes em relação à Matemática (Brito, 1996, 1998; Gonçalez \& Brito, 1996; Lipnevich, Maccann, Krumm, Burrus, \& Roberts, 2011) e a solução de problemas matemáticos (Pajares, 1996; Pajares \& Miller, 1994; Zimmerman \& Campillo, 2003).

A solução de problemas está presente no cotidiano e no contexto acadêmico em geral, sendo uma atividade freqüentemente associada às aulas de Matemática. Pode-se afirmar que um problema é caracterizado por uma situação em que um indivíduo ou um grupo quer ou precisa resolver e para a qual não dispõe de um caminho rápido e direto que o leve à solução (Lester, 1983, citado por Echeverría \& Pozo, 1998, p. 15). Brito (2012, p. 18) apontou que a:

solução de problemas é entendida como uma forma complexa de combinação dos mecanismos cognitivos disponibilizados a partir do momento em que o sujeito se depara com uma situação para a qual precisa buscar alternativas de solução. Pode ser definida como um processo cognitivo que visa transformar uma dada situação em uma situação dirigida a um objetivo, quando um método óbvio de solução não está disponível para o solucionador, apresentando quatro características básicas: é cognitiva, é um processo, é dirigida a um objetivo e é pessoal, pois depende do conhecimento prévio do indivíduo.

Para uma melhor compreensão do processo de solução de problemas matemáticos, é relevante destacar as ideias de Mayer (1998), para quem solucionar um problema requer uma representação do problema, a elaboração de um plano de solução e a execução do plano, ou seja, aplicar as estratégias necessárias para se chegar à solução. Para Mayer (1998) este processo é afetado por aspectos cognitivos, metacognitivos e motivacionais. Dentre os aspectos motivacionais, Mayer se refere ao constructo de autoeficácia, apontando que os estudantes se dedicam mais a uma tarefa quando se julgam capazes, do que quando falta confiança em suas habilidades para aprender.

Alguns estudos (Inglez de Souza, 2006; Stevens et al., 2004; Zimmerman \& Campillo, 2003) têm apontado a autoeficácia como um importante elemento mediador da solução de problemas, afetando a quantidade de tempo, esforço e persistência despendidos por estudantes ao se depararem com tal tarefa. Assim, de acordo com Pajares e Miller (1994) a autoeficácia se sobrepõe a outros fatores preditivos do sucesso nesta atividade, como gênero, autoconceito e percepção de utilidade da Matemática.

A escola possui um papel fundamental no desenvolvimento dos estudantes e um objetivo central é formar bons pensadores (Brito, 2002). Aliado a esse objetivo, a teoria social cognitiva apresenta evidências de que o desenvolvimento de autoeficácia e da capacidade de autorregulação permite ao estudante usar mecanismos de ajuste frente a determinadas tarefas. O ensino de Matemática trabalha em grande parte com tarefas que envolvem a solução, pelos estudantes, de problemas de diferentes tipos. O domínio de um conteúdo escolar envolve também o esforço do estudante para compreender as exigências do problema e colocar em ação os mecanismos cognitivos que permitam alcançar, com sucesso, a solução da tarefa apresentada. As atividades escolares devem ser planejadas tendo em vista as crenças pessoais dos estudantes a respeito da tarefa que precisam realizar, como estes se percebem frente determinada tarefa e suas atitudes em relação àquele conteúdo, pois isso poderá afetar o desempenho do estudante em situações futuras.

A Matemática escolar tem sido objeto de estudos desde a década de quarenta do século passado e as pesquisas abrangem tanto aspectos cognitivos quanto afetivos, sendo que esses úl- 
timos tiveram um incremento maior a partir dos estudos da teoria social cognitiva. A solução de problemas, elemento chave no ensino de Matemática, também tem sido analisada desde longa data e sob vários aspectos, incluindo ai a motivação intrínseca e extrínseca, a ansiedade e as relações desses construtos com a aprendizagem e o desempenho (Skemp, 1971). Uma maneira de auxiliar os estudantes a construir formas eficientes de pensar os problemas com os quais se defrontam e assim adquirir um maior domínio de uma dada área é através da apresentação de tarefas desafiadoras e motivadoras, levando-os ao desenvolvimento do pensamento flexível e produtivo na solução de problemas de diferentes tipos apresentados em diferentes contextos (Brito, 2010, 2011).

As tarefas cognitivas com as quais o estudante se defronta ao solucionar um problema estimulam a curiosidade, levando-os a exercitar a criatividade. Além disso, de acordo com a teoria do processamento humano da informação o pen- samento e os processos cognitivos são seqüenciais, isto é, cada estágio fornece condições para o aparecimento do seguinte.

Frente a um problema, o estudante tem a atenção voltada para as exigências daquela tarefa específica e disponibiliza mecanismos pertinentes à realização da mesma, buscando executá-la com êxito. Nessa busca, em direção à realização da tarefa, os componentes cognitivos, afetivos e motores interatuam de forma orgânica.

Muitos autores se empenharam em descrever as etapas pelas quais passa o pensamento durante a solução de problemas. Brito (2011) apresentou uma revisão desses estudos e apontou as seguintes etapas: (a) compreensão do enunciado ou do texto (história do problema); (b) representação do problema, (c) categorização do problema; (d) estimativa de solução; (e) planejamento da solução; (f) autoavaliação do procedimento utilizado; (g) autoavaliação do cálculo e (h) Redação da resposta, que leva o estudante a uma nova leitura da proposição do problema e compreensão do texto.
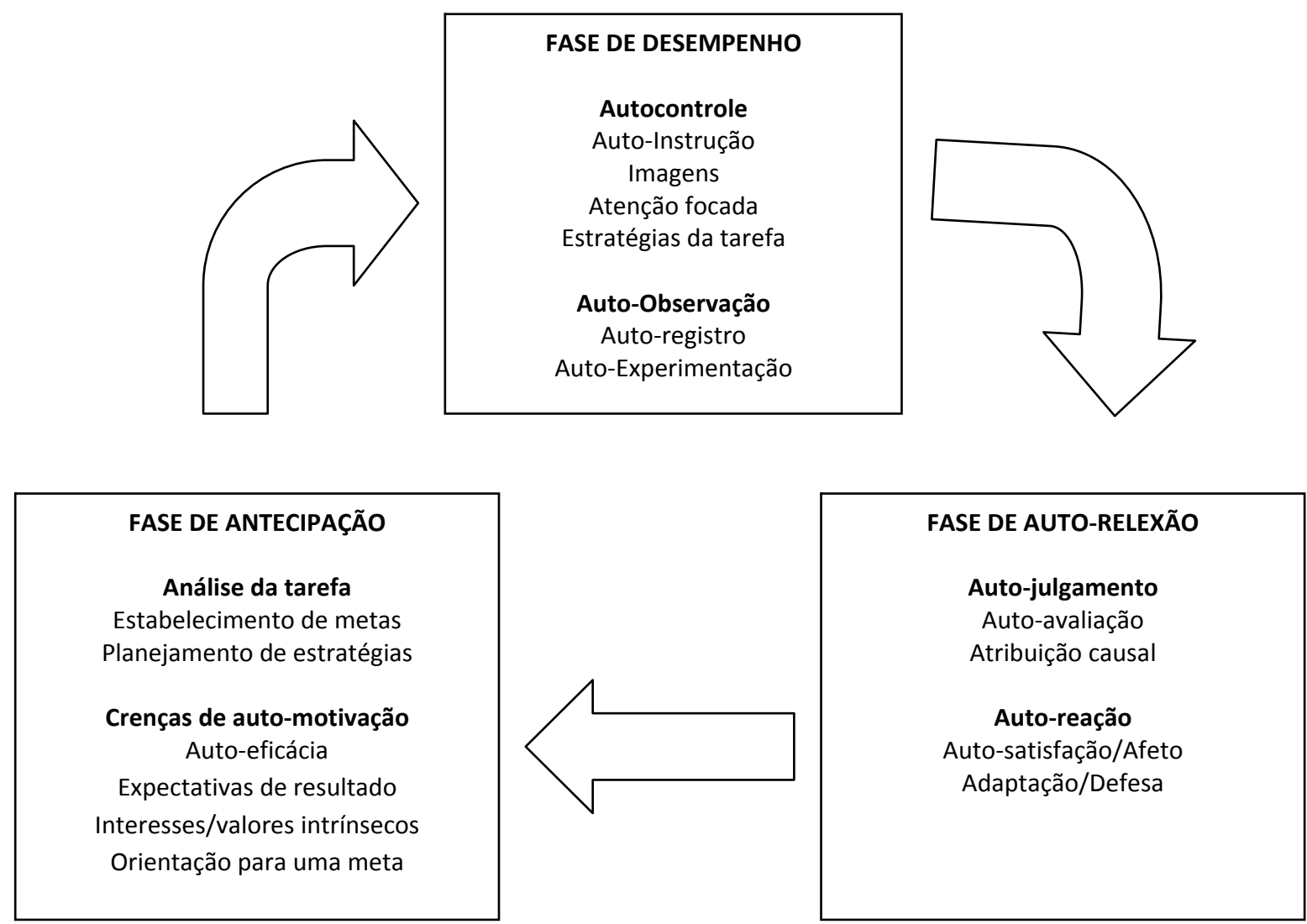

Figura 1. Fases e subprocessos da auto regulação na solução de problemas (adaptado de Zimmerman \& Campillo, 2003; tradução livre das autoras). 
A Figura 1 proposta por Zimmerman e Campillo (2003) mostra três fases da solução de problemas (fase de antecipação, de desempenho e de autorreflexão) e subjacente a cada uma delas, os subprocessos componentes e que são ativados durante a realização da tarefa. As três fases e os correspondentes subprocessos de autorregulação durante a solução de problemas podem ser relacionadas com essas oito etapas de solução mostradas no parágrafo anterior. A parte inicial, quando o indivíduo se situa no "espaço do problema" seria a fase de antecipação incluindo aí a análise da tarefa e o estabelecimento de metas visando atingir o objetivo final. As crenças motivacionais incluem as crenças de autoeficácia, a expectativa de resultado e os interesses e valores intrínsecos, sendo esses orientados para uma meta a ser cumprida. As fases 2, 3, 4 e 5 estariam relacionadas ao autocontrole e auto-observação enquanto 7 e 8 seriam compatíveis com a autorreflexão. Todos esses aspectos estão intimamente entrelaçados e deve ser considerado que a solução de problemas envolve tanto características do problema (por exemplo, um problema que envolve estimativa) quanto aspectos relativos ao solucionador (ter conhecimento sobre estimativa, atitudes em relação à Matemática, motivação para buscar a solução).

Zimmerman e Campillo (2003) apontaram a necessidade de ser observada, pelos educadores, a relação cíclica entre as crenças motivacionais e os processos autorregulatórios e, como exemplo, foi usada a estimativa. $\mathrm{O}$ instrumento de autoeficácia matemática usado no presente estudo apresenta vários problemas de estimativa. Na parte 2, onde aparecem 5 alternativas, o estudante deve escolher apenas uma delas e não existe resposta correta; assim, o solucionador necessita identificar os principais elementos do problema, ser capaz de estimar a resposta e, mesmo intuitivamente, conhecer as regras concernentes à estimativa. Nesse caso, o estudante precisa persistir no problema e ativar mecanismos que permitam a ele determinar o ponto em que o problema termina e isso não ocorre de forma casual. Nas situações escolares a estimativa de solução é planejada e ensinada de modo a levar o estudante a perceber que mesmo em problemas que não apresentam a resposta exata é possível estimar uma solução.

\section{Método}

Esse estudo, que é parte de uma pesquisa mais ampla e que envolve estudantes de várias escolas e de diferentes séries é de caráter descritivo e teve como objetivo avaliar as crenças de autoeficácia para solucionar problemas de Matemática, buscando verificar se existiam diferenças entre as crenças de autoeficácia frente a tarefas específicas e o desempenho efetivo de estudantes da quinta série frente à mesma tarefa (um conjunto de 20 problemas matemáticos) apresentada após um período de tempo. Buscou avaliar também o autoconceito matemático e a autoeficácia para a aprendizagem autorregulada tendo como referencial as fases e subprocessos da auto regulação na solução de problemas propostos por Zimmerman e Campillo (2003).

Tendo em vista que algumas variáveis estão presentes nas etapas de solução de problemas matemáticos levando ao sucesso ou fracasso na tarefa (Brito, 1996, 2010), buscou-se verificar essas variáveis: importância da Matemática, preferência por disciplina e ansiedade matemática, além de gênero, classe e idade. Com o objetivo de medir como as variáveis do estudo estão relacionadas, foi feita uma correlação entre os instrumentos utilizados.

A autoeficácia tem sido considerada um importante elemento preditivo das realizações alcançadas pelos estudantes (Cleary \& Chen, 2009). Tem-se argumentado que o valor preditivo desta crença está vinculado à forma com que é avaliada. Por exemplo, Chen (2003) utilizou o mesmo procedimento adotado na presente pesquisa: os estudantes deveriam atribuir um valor à própria confiança em solucionar cada item da prova de Matemática. Esta escala representa uma avaliação bastante específica à tarefa de como os estudantes percebem sua capacidade matemática. Além disso, deveriam responder a duas escalas, sendo uma delas sobre autoconceito matemático e a outra uma escala de autoeficácia regulada. Alguns outros aspectos como preferência por disciplina que estão relacionados 
ao autoconceito e à autoeficácia também foram identificados.

\section{Participantes}

Os participantes desse estudo foram 131 estudantes da quinta série do período matutino, sendo 72 do gênero masculino e 59 do gênero feminino, matriculados em uma escola confessional privada de uma cidade de grande porte da região sudeste do país. A professora de Matemática era a mesma para as quatro séries e os estudantes estavam distribuídos da seguinte maneira: 32 estudantes nas classes A; B e D e 35 na classe C. A idade desses estudantes variava de 11 anos (87 estudantes $-66,6 \%$ ); 12 anos (40 estudantes $-30,5 \%$ ) e 13 anos (4 estudantes $-3,1 \%$ ) e os demais não informaram a idade. Esse estudo é um recorte de um estudo mais amplo realizado com 603 estudantes de quinta, sexta, sétima e oitava séries. A razão da opção pela quinta série da mesma professora foi a necessidade de isolar as variáveis professora e idade dos sujeitos. Outro fator considerado foi o instrumento usado para aferir a crença de autoeficácia matemática, tendo em vista que cada uma das séries era submetida a um instrumento no qual os problemas eram relativos ao conteúdo tratado naquela etapa escolar.

\section{Procedimento}

Os instrumentos foram aplicados pelas pesquisadoras no horário da aula de Matemática, porém sem a presença da professora, tendo em vista que estudos anteriores (Brito, 1996) mostraram que a presença da professora pode influenciar a resposta dos estudantes quando estes são solicitados a emitir juízos de valor sobre a disciplina, o conteúdo e o desempenho. Como eram muitos instrumentos, os mesmos foram respondidos em dias alternados após o consentimento para participação. No primeiro dia, os participantes respondiam aos seguintes instrumentos: Escala de autoconceito matemático, Escala de atitudes em relação à Matemática, Escala de autoeficácia para aprendizagem autorregulada e Instrumento de Autoeficácia matemática - $5^{\text {a }}$ série- parte 1 . O aplicador era sempre o mesmo em todas as sessões de uma mesma classe; as instruções eram dadas no início da atividade. As perguntas dos estudantes eram respondidas à medida que apareciam e era solicitado a eles que não tentassem se comunicar.

$\mathrm{Na}$ segunda etapa, que ocorreu 15 dias depois, os estudantes foram solicitados a responder aos seguintes instrumentos: Escala de ansiedade matemática e Instrumento de autoeficácia matemática - $5^{\text {a }}$ série - parte 2, além da Escala de importância da Matemática. A aplicação foi feita ao final do quarto bimestre do ano letivo de modo a assegurar que os conteúdos tratados não ocorressem simultaneamente à aplicação.

Finda a coleta de dados, os instrumentos foram agrupados por estudante e por classe e as respostas obtidas foram organizadas em uma base de dados. Em seguida foram efetuadas as notas e médias dos instrumentos utilizados. Todas as escalas usadas no presente estudo foram convertidas para a mesma métrica de modo a permitir a comparação, com notas médias finais variando entre 1 e 8 (Smith, Wakely, de Kruif, \& Swartz, 2003).

O presente estudo apresenta uma análise descritiva dos resultados obtidos em cada um dos instrumentos considerando gênero, idade e preferência por disciplina. Posteriormente são apresentadas as correlações obtidas e as variâncias entre os grupos, considerando o desempenho na solução dos problemas propostos. Os dados resultantes da aplicação dos instrumentos de pesquisa foram analisados por meio do software Statistical Package for Social Science tendo sido estabelecido o nível de significância de 0,05 para a tomada de decisões baseada nas análises estatísticas realizadas.

\section{Instrumentos}

Escala de Autoconceito Matemático. A escala de autoconceito foi construída por Pajares e Miller (1994). Essa escala foi traduzida para o português e validada com estudantes brasileiros tendo obtido um coeficiente alfa de 0,90 (L. F. Neves, 2002; L. F. Neves \& Brito, 2001). É uma escala de tipo Thursthone que apresenta 21 itens com as seguintes alternativas: totalmente falsa (1 ponto); falsa, maior parte falsa, mais falsa que verdadeira, mais verdadeira que falsa, maior parte verdadeira, verdadeira e totalmente verdadeira 
(8 pontos). As proposições versam sobre autoconceito matemático, por exemplo: Em comparação com os meninos da minha classe, eu sou bom em Matemática. Há também itens que versam sobre atitudes, como Eu acho a Matemática interessante. Essa escala busca captar a autopercepção do estudante a respeito da aprendizagem e desempenho em Matemática, solução de problemas, da própria capacidade nessa disciplina e também a percepção de aspectos relacionados ao ambiente e às tarefas propostas. A escala vem precedida de um espaço onde o estudante coloca a identificação, necessária para a comparação posterior: nome, idade, gênero, tipo de escola (pública ou particular), série, período, nome da professora e é solicitado a indicar a disciplina favorita e a disciplina que menos gosta de estudar. Três questões dessa escala apresentam sentimentos negativos ("A Matemática é chata", por exemplo) e na atribuição de pontos é feita uma inversão nesses três itens. A média dessa escala foi expressa no intervalo entre 1 e 8 .

Escala de Atitudes em Relação à Matemática (Metas de Realização). A escala de atitudes em relação à Matemática com vistas ao cumprimento de uma tarefa (no presente caso, uma tarefa matemática) é um instrumento com 20 questões que versam sobre as razões pelas quais os estudantes completam ou não as tarefas matemáticas, como se percebem frente aos outros estudantes na realização de tarefas matemáticas e como participam nas aulas de Matemática. É baseada em uma escala originalmente concebida por Albert Bandura com 6 itens, a qual buscava aferir as metas de realização para a escrita (Zimmerman, Bandura, \& Martinez-Pons, 1992). Foi adaptada para a Matemática por Frank Pajares e traduzida e adaptada para o português pelas autoras. Como muitos itens dessa escala são semelhantes a muitas escalas de atitudes, optou-se por incluir atitudes na designação da escala. Nessa versão, a escala apresenta uma régua com itens de 1 (um) a 8 (oito) pontos e o estudante é instruído a escolher qualquer item entre estes valores. Os itens são: 1- Totalmente falsa; 2- Falsa; 3- Maior parte falsa; 4- Mais falsa que verdadeira; 5- Mais verdadeira que falsa; 6- Maior parte verdadeira; 7- Verdadeira; 8- Totalmente verda- deira. Para a análise as questões com conotação negativa são invertidas e a média é dada com valores entre 1 e 8 .

Escala de Importância da Matemática. A escala de importância da Matemática é composta de 10 afirmações a respeito do grau de importância da Matemática na vida dos indivíduos, não se limitando especificamente a situações escolares. Na instrução é ponderado que as pessoas pensem de forma diferente em relação à importância da Matemática e é solicitado que em uma escala de 1 a 8 (extremamente sem importância, pouco importante, moderadamente importante, muito importante e extremamente importante). Os estudantes são solicitados a se posicionar sobre a importância da Matemática em relação a itens amplos como "Ter uma vida feliz" e "Ser um bom cidadão" a mais específicos como "Arrumar um emprego" ou "Formar-se em uma faculdade".

Escala de Autoeficácia para Aprendizagem Autoregulada. Esta é uma subescala com sete itens utilizada por Pajares e Graham (1999), que foi elaborada e validada a partir da Escala Multidimensional de Autoeficácia de Crianças de Bandura (Zimmerman et al., 1992). A escala original de Bandura era composta de onze itens que foram reduzidos para sete proposições por Pajares e Graham (1999). São esses sete itens que foram traduzidos e adaptados para o presente estudo que compõem a Escala de autoeficácia para Aprendizagem autorregulada. Esta escala avalia os julgamentos que os alunos fazem a respeito da própria capacidade de utilizar diversas estratégias de autorregulação da aprendizagem, como estudar com atenção, terminar os trabalhos de casa no prazo, planejar e organizar as atividades escolares. Trata-se de uma escala que varia de 1 (um) a 8 (oito) pontos, com os 8 pontos assinalados em uma linha horizontal e quatro itens avaliativos (dificilmente, com alguma dificuldade, mais ou menos, com alguma facilidade, facilmente) que permitem ao sujeito escolher a posição em que estaria situado em relação a cada uma das proposições.

Escala de Ansiedade Matemática. Este instrumento refere-se a uma adaptação por Betz (1978) da Escala de Ansiedade de Fennema e 
Sherman e adaptada por Pajares e Miller (citados por Pajares \& Miller, 1994) e contém nove itens versando sobre ansiedade relacionada à Matemática. Os itens também são respondidos através de escala tipo Thurstone, de oito pontos, variando de "totalmente falsa" a "totalmente verdadeira". Muitos itens são negativos e, portanto têm pontuação invertida, por exemplo: $E u$ me apavoro quando tenho que estudar Matemática. $\mathrm{O}$ estudante que assinala 1 nessa afirmação recebe 8 pontos após a inversão. A média nessa escala, assim como nas demais, varia de 1 a 8 . Desta forma, quanto maior a pontuação obtida pelo sujeito, menor sua ansiedade relativa à Matemática, pois ele assinalou mais itens positivos de apreciação da Matemática.

Instrumento de Autoeficácia Matemática $5^{a}$ Série - Parte 1 e Parte 2. O instrumento, tipo lápis e papel, utilizado para medir a autoeficácia matemática foi traduzido e adaptado do instrumento utilizado por Pajares e Graham (1999) e é composto de 20 problemas envolvendo conhecimentos de álgebra e aritmética que constam da proposta curricular de Matemática da quinta série. Os estudantes são solicitados a expressar o grau de confiança para resolver 20 problemas de Matemática e respondem o instrumento em dois momentos: Em um primeiro momento é apresentada apenas a proposição do problema e os estudantes devem apenas ler e assinalar, em uma escala de oito pontos (onde 1 era nada confiante e 8 totalmente confiante), qual o grau de confiança de que seriam capazes de solucionar cada um dos problemas. A instrução que vem no início do instrumento era a seguinte:

Suponha que amanhã você seja solicitado a solucionar os seguintes problemas de Matemática, contidos em uma prova com questões de múltipla escolha. Por favor, leia atentamente os problemas e indique o grau de confiança que você tem de que será capaz de acertar o problema, sem o uso de calculadora. Por favor, não tente resolver o problema, mas lembre-se que você deve ler as questões e responder como se fosse para uma prova em sala de aula.

Em seguida são apresentados os 20 problemas que foram traduzidos e adaptados para o presente estudo. Em frente aos problemas aparecem os números de 1 a 8 e os estudantes devem responder de acordo com o grau de confiança que têm de que acertarão aquela questão. Exemplos de problemas: Um motorista pretende viajar $1900 \mathrm{~km}$ desde Campinas até Aracaju. No primeiro dia, ele dirigiu $525 \mathrm{~km}$. Calcule quantos quilômetros faltam para o fim da viagem. $\mathrm{Na}$ frente do problema apareciam as opções 123 45678 e o estudante, baseado na régua que aparecia no início da folha, assinalava onde se encontrava o grau de confiança de que solucionaria o problema.

Quinze dias depois os estudantes eram submetidos a um segundo instrumento com os mesmos problemas (Instrumento de Autoeficácia Matemática $-5^{\mathrm{a}}$ Série - Parte 2). Porém, desta feita eram apresentadas cinco alternativas de resultado e os estudantes deveriam assinalar a alternativa correta ou aquela que mais se aproximava da correta, dando a resposta do problema. Alguns problemas envolviam estimativa de cálculo e outros problemas envolviam aproximação para centena ou milhar. Um exemplo dos problemas do $2^{\circ}$ instrumento é apresentado a seguir:

Um motorista pretende viajar 1900 quilômetros desde Campinas até Aracaju. No primeiro dia, ele dirigiu 525 quilômetros. Calcule quantos quilômetros faltam para o fim da viagem.
(a) 675
(b) 1230
(c) 1377
(d) 21
(e) 1525

No primeiro instrumento, ao defrontar-se com o problema, o estudante avalia a expectativa de realizar uma tarefa específica com sucesso. De acordo com a teoria social cognitiva, através da autoavaliação da tarefa é possível predizer o desempenho efetivo do estudante. Nos dois instrumentos, ao final da apresentação dos problemas, era solicitado ao estudante que informasse qual a nota (em uma escala de zero a dez) que ele acreditava que tiraria se aquilo fosse realmente uma prova e também qual a porcentagem de acertos que ele teria em uma escala de 1 a 100 .

Os pontos foram atribuídos de 20 a 160 e o resultado foi dividido pelo número de questões 
(20) de forma a gerar uma nota média variando de 1 a 8 , coerente com os demais instrumentos usados.

Ao final do Instrumento de Autoeficácia Matemática - $5^{\text {a }}$ Série - Parte 1 e Parte 2, os estudantes eram solicitados a fazer uma análise pré- via do desempenho, pois isso é mais uma informação sobre as crenças que o estudante tem na própria capacidade frente aos problemas apresentados. Na segunda parte, a expressão "já leu" foi substituída por "já resolveu". A solicitação era a seguinte:

Agora que você já resolveu todos os 20 problemas, quanto (em porcentagem) você acha que poderia acertar desse teste, se ele fosse uma prova de múltipla escolha? Assinale quanto você acha que acertaria na linha abaixo:

\begin{tabular}{lllllllllll}
$0 \%$ & $10 \%$ & $20 \%$ & $30 \%$ & $40 \%$ & $50 \%$ & $60 \%$ & $70 \%$ & $80 \%$ & $90 \%$ & $100 \%$ \\
\hline & & & & & & & & & & \\
\hline
\end{tabular}

Se fosse dada uma nota de zero a dez $(0$ - 10), eu tiraria (complete com a nota que você acha que tiraria).

\section{Resultados}

Os sujeitos foram 131 estudantes da quinta série sendo 72 do gênero masculino e 59 do gênero feminino, com idades variando entre 11 e 13 anos. Desses 131 estudantes, 25 deles apontam a Matemática como a disciplina favorita $(19,1 \%)$; 16 indicam Ciências (12,2\%); 15 escolheram Educação Artística (11,5\%); 11 apontam História $(8,4 \%) ; 11$ estudantes escolheram Educação Física $(8,4 \%)$ e os demais escolheram entre as outras disciplinas. Quando perguntados sobre a disciplina que menos gostam, 47 afirmaram ser Português $(35,9 \%)$ e 33 indicaram a disciplina Geografia (25,2\%); 13 alunos (9,9\%) apontaram a Matemática e os demais se distribuíram nas outras opções.

A primeira pergunta apresentada aos estudantes era se gostavam de resolver problemas matemáticos difíceis e eles se distribuíram da seguinte forma: 2 não responderam $(1,5 \%) ; 50$ $(39,7 \%)$ afirmaram ser falsa a proposição, indicando que não gostavam de resolver problemas difíceis e 79 alunos (60,3\%) afirmaram gostar, em maior ou menor grau, de solucionar problemas matemáticos difíceis. Mas, dos estudantes que afirmaram gostar, apenas 16 deles escolheram a alternativa totalmente verdadeira.

A média na Escala de Auto Conceito Matemático foi 5,655 $(D P=1,1512)$ variando entre 2,5 e $7,9(n=128)$. Não foram encontradas diferenças significativas que pudessem ser atribuídas ao gênero, à série e à idade, embora os estudantes com 12 anos tenham obtido as médias menores. Também não foram encontradas diferenças significativas nas médias entre as quatro classes. Quando os estudantes foram separados de acordo com a preferência por disciplina são verificadas diferenças significativas $(p=0,001)$, sendo que a maior média é do grupo $(n=24)$ que aponta a Matemática como a disciplina favorita (média $=6,609 ; D P=0,6662$ ). Houve apenas um aluno que apontou não ter preferência por nenhuma disciplina escolar (média $=6,286$ ), e, em seguida, aqueles $(n=16)$ que preferem Ciências (média $=6,202 ; D P=0,7664$ ). Por outro lado, quando são agrupados de acordo com a disciplina que menos gostam, pode ser observado que os estudantes $(n=13)$ que apontaram a Matemática como a disciplina que menos gostam são aqueles com a média mais baixa (média $=4,795$; $D P=1,2044)$. Apenas os dois estudantes que informaram não gostar de nenhuma disciplina apresentaram média mais baixa (média $=3,667$; $D P=1,3469$ ). Esses resultados são coerentes com outros realizados a respeito das atitudes em relação à Matemática, pois existe estreita relação entre as atitudes em relação à Matemática e o autoconceito matemático, isto é, estudantes com autoconceito matemático desfavorável, estão propensos a atitudes negativas em relação à Matemática (Dobarro \& Brito, 2010).

A média na Escala de Atitudes em Relação à Matemática (Metas de Realização) foi 5,530 $(D P=1,0772)$ com pontuação variando entre 2,7 e 7,9 . Foi verificado que não existiam dife- 
renças significativas entre as médias na escala quando comparadas as quatro classes. Também não foram encontradas diferenças significativas quando agrupados de acordo com a idade e a preferência por disciplina. Quando os estudantes foram agrupados de acordo com o gênero, os meninos $(n=59)$ obtiveram média igual à 5,704 e as meninas $(n=58)$ média igual a 5,322 . Foram encontradas diferenças significativas entre os dois grupos $(F=4,058 ; p=0,046)$, indicando que as metas de realização das meninas desse grupo são inferiores aos meninos e as atitudes mais negativas.

$\mathrm{Na}$ Escala de Importância da Matemática a média entre estes estudantes foi 6,660 $(n=131$; $D P=1,2125)$. Quando os estudantes são agrupados de acordo com o gênero, aparecem diferenças significativas em relação à média $(p=0,046$; $F=4,064)$ sendo que a média dos meninos é 6,851 e as meninas $m=6,427$, indicando uma pequena, porém significativa diferença à favor dos meninos na importância que esse grupo atribui à Matemática. Também aparecem diferenças significativas entre os grupos, quanto à idade $(F$ $=3,907 ; p=0,023$ ) sendo os alunos mais novos (11 anos, $n=84$ ) aqueles que atribuem um grau maior de importância à Matemática.

Quando agrupados de acordo com a classe e preferência por disciplina não foram encontradas diferenças significativas. Foi observado que o limite mínimo na escala de importância atribuída à Matemática foi 3,1 indicando que os estudantes desse grupo, de maneira geral, percebem a relevância desta disciplina diferindo no grau que essa importância assume.

A média na Escala de Autoeficácia para Aprendizagem Autorregulada foi $m=5,871$ (DP $=1,5102 ; n=131)$ e não foram encontradas diferenças significativas quando os estudantes foram agrupados de acordo com o gênero, a classe, idade e disciplina que menos gostam. Quando os estudantes são agrupados de acordo com a disciplina preferida, aparecem diferenças significativas entre os grupos $(p=0,01)$. Os estudantes que preferem Matemática, Ciências e Geografia foram os que obtiveram as maiores médias.

A média na Escala de Ansiedade Matemática foi $5,103(n=131 ; D P=1,7391$; mínimo
$=0,0$ e máximo $=8,0)$. Não foram encontradas diferenças significativas quando os estudantes são agrupados por gênero, classe e idade. Porém, apareceram diferenças significativas quando os estudantes foram agrupados de acordo com as disciplinas preferidas e aquelas que menos gostam, sendo que os estudantes que preferem a Matemática não apresentam ansiedade Matemática ou esta é muito mais baixa.

No Instrumento de Autoeficácia Matemática $-5^{\text {a }}$ Série - Parte 1, quando os estudantes apenas apontavam quanto acreditavam que obteriam em uma prova com aqueles problemas, em uma escala de 1 a 8 foi obtida a média 5,91 ( $n=$ $130 ; D P=1,2125)$. Quando os estudantes foram agrupados de acordo com o gênero, a idade e a preferência por disciplina, não foram encontradas diferenças significativas entre as médias dos estudantes. Foram encontradas diferenças significativas entre as quatro classes $(F=3,989 ; p=$ $0,009 ; n=129)$ o que indica que duas classes apresentam médias significativamente maiores que as outras duas.

Nesta primeira parte, quando perguntados sobre a porcentagem de acerto que acreditavam que teriam em uma prova com esse conjunto de problemas, a porcentagem média obtida foi $66,5 \%$ (variando de 0 a $100 \%$ ) e não foram encontradas diferenças de gênero e preferência por disciplina, mas foram encontradas diferenças quando os estudantes foram agrupados pelas classes ( 4 grupos, $n=130 ; F=4,386 ; p=0,006$ ) e pela idade ( 3 grupos, $n=130 ; F=4,032 ; p=$ 0,020 ).

Em seguida, os estudantes foram solicitados a indicar uma nota (entre zero e dez) que acreditavam que poderiam obter, caso aqueles problemas constituíssem uma prova escolar. A média do grupo foi 7,87 $(n=111 ; D P=1,452)$. Quando considerado o gênero dos participantes, foi verificado que as médias foram muito próximas, sendo $7,86(n=60 ; D P=1,380)$ a média dos meninos e $7,88(n=51 ; D P=1,542)$ a média das meninas, não tendo sido verificada diferença significativa entre os dois grupos. Também não apareceram diferenças significativas quando os estudantes foram agrupados por classe e por idade. As diferenças significativas ( $p=$ 
$0,23)$ se fizeram presentes quando os estudantes foram agrupados de acordo com a preferência por disciplina (média $=7,87 ; D P=1,452$ ), sendo que os estudantes que preferem Matemática obtiveram média $=8,79(D P=0,943)$ e os que preferem Ciências tiveram média $=8,46(D P=$ $0,843)$.

No Instrumento de Autoeficácia Matemática - $5^{\text {a }}$ Série-Parte 2 houve um problema (questão 11) anulado porque a alternativa de resposta foi digitada incorretamente. Assim, as notas poderiam variar de 0,0 (zero) a 9,5 (nove e meio) visto que cada questão correta recebia 0,5 pontos. Nos problemas de estimativa, não existia uma alternativa com o valor exato porque o objetivo era que o estudante e encontrasse o valor de acordo com as regras da Matemática. Muitos solucionaram o problema e colocaram a resposta encontrada adicionando uma alternativa f. Embora muitos acertassem o problema a resposta não foi considerada correta porque desviava do objetivo das questões que versavam sobre estimativa.

A média obtida pelos participantes neste instrumento foi $6,120(D P=1,7831 ; n=121$; sendo que os meninos obtiveram média 5,606 $(D P=1,9085)$ e as meninas, média 6,736 $(D P=$ $1,4039)$, tendo sido encontradas diferenças significativas $(F=13,290 ; p=0,001)$ entre esses dois grupos.

Com relação às diferentes classes $(n=121)$, as médias encontradas foram as seguintes: Classe A: média 5,783 ( $D P=1,9638$; mínima $=2,0$ e máxima $=9,5 ; n=30$ ); Classe B: média 6,823 $(D P=1,6204 ;$ mínima $=2,0$ e máxima $=8,5 ; n=$ 31); Classe C: média 6,276 ( $D P=1,6775$; mínima $=2,5$ e máxima $=9,0 ; n=29)$; Classe D: média 5,597 $(D P=1,6754$; mínima $=2,5$ e máxima $=8,0 ; n=29)$. Foi possível identificar diferenças significativas entre esses grupos $(p=0,030)$.

Não foram encontradas diferenças significativas quando os estudantes foram agrupados de acordo com a idade e com a preferência por disciplina, sendo que os 24 estudantes que informaram preferir a Matemática obtiveram média $6,625(D P=1,8361)$. Esta média é inferior à media obtida pelos 16 estudantes que afirmaram preferir ciências (média $=6,813 ; D P=1,5152$ ) não tendo sido encontradas diferenças significativas entre os grupos.

Quando perguntados sobre a porcentagem de acertos que acreditavam que teriam, a porcentagem média foi $75 \%$ de acertos sendo que as meninas estimaram uma porcentagem média de acertos de $76,67 \%$ e os meninos calcularam que acertariam $73,76 \%$, não havendo diferença significativa entre os grupos. Também não foram encontradas diferenças significativas quando os estudantes foram separados por idade e classe. Quando os estudantes foram separados de acordo com a preferência por disciplina, foram verificadas diferenças significativas $(F=3,382 ; p$ $=0,01)$ nas médias das porcentagens de acerto que acreditavam que teriam: os estudantes que preferem a Matemática acreditavam que teriam $88,5 \%$ de acerto; os que indicaram Ciências como disciplina preferida, afirmaram acreditar que acertariam $85 \%$ da prova e os que preferiam Educação Física, acreditavam que acertariam $81 \%$ da prova.

A nota que os estudantes se atribuíam ao final da solução dos problemas do instrumento 2 , variando de zero a dez, teve média 7,991 ( $D P$ $=1,7768 ; n=106)$, sendo que os 57 estudantes do gênero masculino tiveram média 7,825 ( $D P$ $=1,8904)$ e as 49 estudantes do gênero feminino obtiveram média 8,184 $(D P=1,6327)$. Porém, essas diferenças não são significativas, embora o desempenho das meninas seja ligeiramente superior ao dos meninos. Também não foram encontradas diferenças significativas nas notas que os estudantes se auto-atribuiam quando foram agrupados por classe. Porém, se compararmos esses resultados por classe com a nota real de cada uma das classes, é possível verificar que os estudantes acreditavam que o desempenho seria muito superior ao efetivamente observado. Não foram encontradas diferenças significativas quando os estudantes foram agrupados por idade. Quando agrupados de acordo com a preferência por disciplina foram encontradas diferenças significativas próximas do valor estipulado $(p=0,051)$ como intervalo de confiança. Os 21 estudantes que afirmaram ser a Matemática a disciplina preferida obtiveram as maiores notas e média $8,824(D P=1,1882)$, seguidos daqueles 
que indicaram Ciências (média $=8,613 ; D P=$ 1,2312).

Em seguida, com o objetivo de verificar se as variáveis estavam relacionadas, foi efetuada a correlação de Pearson entre os instrumentos usados. A relação entre a escala de autoconceito matemático e a escala de atitudes em relação à Matemática (metas de realização) é linear positiva e significativamente diferente de zero, $r(124)=0,403 ; p<0,001$, indicando que quanto maiores as pontuações na escala de autoconceito matemático maiores tendem a ser as pontuações na escala de atitudes em relação à Matemática (metas de realização). Em outras palavras, estudantes com elevada crença de autoeficácia tendem a atitudes mais positivas e clareza quanto ao estabelecimento de metas relacionadas a esta disciplina.

Quando são analisadas a escala de auto conceito e a escala de importância da Matemática é encontrada correlação linear positiva e diferente de zero; porém é uma correlação baixa, $r(124)$ $=0,318 ; p<0,001$. O mesmo ocorre com a escala de atitudes (metas de realização) e a escala de importância da Matemática, em que foram encontrados valores baixos, mas ainda significativos, $r(127)=0,246 ; p<0,001$. Esses três instrumentos apresentam pontos distintos, pois enquanto a escala de autoconceito e a de atitudes se referem, quase que na totalidade, a situações da disciplina Matemática no contexto escolar, a escala da importância da Matemática é ampla e trata da Matemática fora do âmbito escolar.

A escala de autoconceito matemático mostrou relação linear positiva e significativamente diferente de zero com todos os demais instrumentos utilizados no presente estudo, sendo que as correlações que apresentaram maiores índices foram entre autoconceito e autorregulação para o estudo, $r(128)=0,508 ; p<0,001$, bem como autoconceito e ansiedade Matemática, $r(128)=$ 0,$556 ; p<0,001$. Isso indica que o estudante que obtém uma maior pontuação na escala de auto conceito tem uma tendência a obter uma pontuação maior na escala de autorregulação para o estudo, além de apresentarem menos ansiedade.

Também foi verificada correlação linear positiva diferente de zero, $r(131)=0,555$; $p<0,001$, entre a escala de autoeficácia para a aprendizagem regulada e a escala de ansiedade Matemática. Trata-se de uma relação moderada que indica que os estudantes que apresentam valores altos na escala de autorregulação apresentam também valores que indicam baixa ansiedade matemática. A escala de autoeficácia matemática - parte 1 e a escala de ansiedade matemática também apresentaram correlação positiva, $r(130)=0,482 ; p<0,001$, e pode ser inferido que, moderadamente, aqueles que obtém maiores resultados para auto-eficácia poderão ter resultados menores de ansiedade.

Finalizando, foi utilizada a correlação de Pearson entre as pontuações em todos os instrumentos utilizados e as notas que os estudantes acreditavam que obteriam e, em seguida, com a nota efetivamente obtida nas tarefas de solução de problemas (nota real).

Os estudantes haviam sido solicitados, em dois momentos, a atribuir uma nota entre 0 e 10 para o desempenho que teriam nos 20 problemas apresentados. Em um primeiro momento, o estudante apenas lê o problema e não tem possibilidade de solução, ficando restrito à fase de antecipação proposta por Zimmerman e Campillo (2003). Nessa fase de antecipação, o estudante analisa mentalmente a tarefa, estabelecendo metas e planejando as estratégias que usará para atingir o objetivo. Em seguida, entram em ação as crenças de motivação que incluem a autoeficácia, as expectativas de resultado, os interesses e valores intrínsecos e a orientação para a meta a ser atingida. São esses sub-processos que o instrumento tenta captar no primeiro momento e, no segundo momento, quando o estudante efetivamente soluciona o problema e escolhe uma alternativa de resposta, os demais sub-processos são efetivados. É importante notar que é possível que, apenas visualizando o problema, a maioria atingisse o sub-processo seguinte (fase de desempenho) usando computação mental, mas não era possível encontrar uma alternativa de escolha na primeira etapa.

A relação entre a escala de autoconceito matemático e a nota que o estudante acredita que obterá é linear positiva e significativamente diferente de zero, $r(108)=0,591 ; p<0,001$. Da 
mesma forma, uma correlação linear positiva diferente de zero foi encontrada entre a escala de autoeficácia para a aprendizagem regulada e a nota que o estudante acredita que terá, $r(110)$ $=0,609 ; p<0,001$, indicando que quanto maior as pontuações na escala de autoconceito matemático e na escala de autoeficácia para a aprendizagem regulada maior tende a ser a nota que o estudante acredita que obterá. Já na escala de atitudes em relação à matemática (metas de realização), embora exista correlação diferente de zero, o valor é bastante baixo, $r(108)=0,225 ; p$ $<0,005$.

A correlação de Pearson também foi calculada para o instrumento de autoeficácia matemática- $5^{\mathrm{a}}$ série-parte 1 e as notas atribuída aos estudantes na solução desses problemas e o resultado mostrou uma correlação linear positiva diferente de zero, $r(120)=0,458 ; p<0,001$, que é uma correlação moderada, indicando que aqueles que obtém pontuações maiores na escala de autoeficácia matemática tendem a obter notas maiores nos problemas. Esse resultado é uma indicação de associação entre o desempenho na solução de problemas e as crenças de autoeficácia dos estudantes desse grupo.

A correlação entre a nota que acredita que obterá e a nota real apresentou correlação diferente de zero, $r(103)=0,501 ; p<0,001$, indicando que aqueles que informaram que tinham expectativas de terem notas altas, efetivamente obtiveram um melhor desempenho. Também foram encontradas associações lineares significativas e diferentes de zero entre a nota real e a escala de autoconceito matemático, $r(119)=0,331$; $p<0,001$; nota real e escala de autoeficácia para a aprendizagem regulada, $r(121)=0,305 ; p<$ 0,001 ; nota real e a escala de ansiedade matemática, $r(121)=0,346 ; p<0,001$. Embora os valores encontrados sejam baixos, indicam algum grau de associação entre as variáveis.

Não foi verificada associação linear significativa entre a nota real dos estudantes e a pontuação na escala de atitudes em relação à Matemática (metas de realização; $r[117]=-0,042$; $p=0,654$ ) e também não foi encontrada entre a nota real e a escala de importância da Matemática, $r(121)=-0,026 ; p<0,779$, indicando que as pontuações são independentes, não sendo possível, nesse grupo, apontar relações entre essas variáveis.

\section{Discussão}

A análise dos dados, através do coeficiente de correlação de Pearson, permitiu fazer algumas inferências quanto à existência de relações entre as variáveis estudadas. Foi possível notar uma relação positiva e estatisticamente significativa entre as pontuações na escala de autoeficácia matemática e as notas obtidas na prova realizada posteriormente $(r=0,458)$. Da mesma forma, as notas obtidas na prova se correlacionaram às pontuações na escala de autoeficácia para a aprendizagem regulada $(r=0,305)$. Neste sentido, é possível afirmar que quanto mais elevadas foram as pontuações nos instrumentos destinados a avaliar a crença de autoeficácia, melhores os resultados na segunda parte da prova que envolvia a efetiva solução dos problemas.

No presente estudo, a ansiedade Matemática mostrou relação inversa com as crenças de autoeficácia, concordando com os resultados obtidos por Bandura (1993, 1995/1999) em que os estudantes com crenças de autoeficácia mais elevadas, apresentavam menor ansiedade. Também são condizentes com os resultados encontrados por Bandalos et al. (1995), que apontaram relação inversa entre autoeficácia e ansiedade frente às avaliações através de provas e exames. Foi verificado também que os estudantes que apontam a Matemática e, em seguida as Ciências como disciplinas preferidas não apresentam ansiedade matemática ou apresentam-na em níveis muito mais baixos. Já os estudantes que preferem disciplinas das áreas de Humanas, foram os que obtiveram médias mais baixas na escala de autoeficácia matemática e foram também os que obtiveram resultado mais indicativos de ansiedade matemática concordando com estudos anteriores (Brito, 1996, 1998).

Os resultados relativos ao gênero foram significativos em alguns instrumentos: importância da Matemática, sendo que os meninos atribuem maior importância à Matemática que as meninas, confirmando resultados anteriores (Gonçalez \& 
Brito, 1996); escala de autoconceito matemático e escala de atitudes em relação à Matemática, mostrando os resultados das meninas inferiores aos dos meninos. Embora muitos estudos mostrem relação entre gênero e ansiedade, nesse grupo não foram encontradas diferenças significativas.

$\mathrm{O}$ gênero feminino mostrou notas maiores na escala de autoeficácia matemática, bem como na tarefa de solução de problemas, sendo que a diferença das médias dos dois grupos foi significativa, mostrando um desempenho superior por parte das meninas. Na atribuição de notas, as meninas atribuem para si notas maiores que os meninos, estando a nota atribuída muito próxima da nota real, indicando, para os sujeitos desse grupo, que as meninas apresentavam crenças diferenciadas a respeito do próprio desempenho (representado pela nota), quando comparadas aos meninos. Pesquisas que controlem melhor variáveis como o domínio do conteúdo (variável cognitiva) na solução de problemas e as crenças de autoeficácia matemática em estudantes da quinta série poderiam explicar melhor essa interação.

No presente estudo, a professora era a mesma para as quatro classes, porém no instrumento de autoeficácia matemática - $5^{\mathrm{a}}$ série-parte 2 , bem como no desempenho (nota) foi encontrada diferença significativa entre as classes.

De um modo geral, os resultados do presente estudo mostraram que a preferência por disciplina (alunos que preferem Matemática e Ciências), as crenças de autoeficácia, o autoconceito e o desempenho na solução de problemas matemáticos estão inter-relacionados e interatuam da maneira mostrada por Zimmerman e Campillo (2003). Concordando com algumas pesquisas baseadas na teoria social cognitiva (Bong, 2004; Dobarro \& Brito, 2010; Inglez de Souza \& Brito, 2008; Pajares \& Miller, 1994) nesse estudo também foi verificada a importância das variáveis motivacionais como preditoras do desempenho (Bandura, 1986, 1995, 1997).

A solução de problemas é uma atividade que ocorre em praticamente todas as aulas de Matemática e com estudantes de diferentes idades; os estudantes são solicitados a resolver problemas individualmente ou em grupo, na sala de aula ou nas tarefas de casa. Ao planejar essas atividades o professor precisa estar atento para alguns aspectos relevantes dessa atividade particularmente com relação à organização das tarefas, que devem ser motivadoras e desafiadoras. Além disso, não se deve perder de vista que as informações fornecidas, pelo professor, acerca do desempenho do aluno, são relevantes na formação das crenças autorreferenciadas, como autoconceito e autoeficácia, sendo relevante o papel desempenhado pelas crenças de autoeficácia e o reconhecimento do desempenho (Stirin, Ganzach, Pazy, \& Eden, 2012).

Muitas vezes toda a atenção do professor é voltada aos processos cognitivos, deixando de lado os processos motivacionais, os processos afetivos e os processos de seleção e são esses que, de certa forma, dirigem a atividade, mobilizando os mecanismos necessários para a ação.

Dada a importância dos aspectos motivacionais no desempenho em solução de problemas e na aprendizagem em geral, é importante reafirmar o papel dos educadores na formação de crenças motivacionais, possibilitando ao aluno desenvolver autopercepções mais favoráveis que sustentem o interesse, a persistência e o envolvimento nos processos de aprendizagem.

\section{Referências}

Arens, A. K., Craven, R. G., Yeung, A. S., \& Hasselhorn, M. (2011). The twofold multidimensionality of academic self-concept: Domain specificity and separation between competence and affect components. Journal of Educational Psychology, 103(4), 970-981. doi: $10.1037 / \mathrm{a} 0025047$

Bandalos, D. L., Yates, K., \& Thorndike-Christ, T. (1995). Effects of math self-concept, perceived self-efficacy, and attributions for failure and success on Test Anxiety. Journal of Educational Psychology, 87(4), 611-623. doi:10.1037/00220663.87.4.611

Bandura, A. (1977). Social learning theory. Englewood Cliffs, NJ: Prentice Hall.

Bandura, A. (1986). Social foundations of thought and action: A social cognitive theory. Englewood Cliffs, NJ: Prentice Hall. 
Bandura, A. (1993). Perceived self-efficacy in cognitive development and functioning. Educational Psychologist, 28(2), 117-148. doi:10.1207/ s15326985ep2802_3

Bandura, A. (1995). Self-efficacy in changing societies. Cambridge, UK: University Press.

Bandura, A. (1997). Self-efficacy: The exercise of control. New York: Freeman.

Bandura, A. (1999). Auto-eficacia: cómo afrontamos los cambios de la sociedad actual (J. Aldekoa, Trad.). Bilbao, España: Desclée de Brower. (Original publicado em 1995)

Barros, A. (1996). Atribuições causais e expectativa de controlo na realização matemática. Psychologica, 15, 135-146.

Betz, N. E. (1978). Prevalence, distribution, and correlates of math anxiety in college students. Journal of counseling Psychology, 25, 441-448.

Bong, M. (2004). Academic motivation in self-efficacy, task-value, achievement goa orientations, and attributional beliefs. Journal of Educational Research, 97(6), 287-297. doi:10.3200/ JOER.97.6.287-298

Brito, M. R. F. (1996). Um estudo sobre as atitudes em relação à matemática em estudantes de $1^{\circ} \mathrm{e}$ $2^{\circ}$ graus (Tese de livre docência não-publicada, Universidade Estadual de Campinas, SP, Brasil).

Brito, M. R. F. (1998). Adaptação e validação de uma escala de atitudes em relação à Matemática. Zetetiké, 6(9), 109-162.

Brito, M. R. F. (2002, abr.). A Psicologia Educacional e a formação do professor-pesquisador: Criando situações desafiadoras para a aprendizagem e o ensino da Matemática [Número especial]. Educação Matemática em Revista (São Paulo), 9(11), 57-68.

Brito, M. R. F. (2010). Alguns aspectos teóricos e conceituais da solução de problemas matemáticos. In M. R. F. Brito (Ed.), Solução de problemas e a Matemática escolar (2. ed., Cap. 1). Campinas, SP: Alínea.

Brito, M. R. F. (2011). Psicologia da Educação Matemática: Um ponto de vista [Número especial]. Educar em Revista, 29-45.

Brito, M. R. F. (2012). É possível avaliar a aprendizagem com o modelo ENADE? Estudos (Brasília), 40, 156-167.

Chen, P. P. (2003). Exploring the accuracy and predictability of the self-efficacy beliefs of seventh- grade mathematics students. Learning and Individual Differences 14, 79-92. doi:10.1016/j. lindif.2003.08.003

Cleary, T. J., \& Chen, P. P. (2009). Self-regulation, motivation, and math achievement in middle school: Variations across grade level and math context. Journal of School Psychology, 47, 291314. doi:10.1016/j.jsp.2009.04.002

Dobarro, V. R., \& Brito, M. R. F. (2010). Atitude e crença de autoeficácia: Relação com o desempenho. Educação Matemática em Revista (São Paulo), 12, 199-220.

Echeverría, M. P., \& Pozo, J. I. (1998). Aprender a resolver problemas e resolver problemas para aprender. In J. I. Pozo (Ed.), A solução de problemas: Aprender a resolver, resolver para aprender (B. A. Neves, Trad.). Porto Alegre, RS: Artmed.

Gonçalez, M. H., \& Brito, M. R. F. (1996). Atitudes (des)favoráveis em relação à matemática. Zetetiké, 4(6), 45-63.

Hackett, G., \& Betz, N. E. (1989). An exploration of the mathematics self-efficacy/mathematics performance. Journal for Research in Mathematics Education, 20(3), 261-273. doi:10.2307/749515

Inglez de Souza, L. F. N. (2006). Crenças de autoeficácia matemática. In R. G. Azzi \& S. A. J. Polydoro (Eds.), Auto-eficácia em diferentes contextos (pp. 111-126). Campinas, SP: Alínea.

Inglez de Souza, L. F. N. (2008). Auto-regulação da aprendizagem e a Matemática escolar (Tese de doutorado em Educação, Faculdade de Educação, Universidade Estadual de Campinas, SP, Brasil).

Inglez de Souza, L. F. N., \& Brito, M. R. F. (2008). Crenças de auto-eficácia, autoconceito e desempenho em matemática. Estudos de Psicologia (Campinas), 25(2), 193-201. doi:10.1590/ S0103-166X2008000200004

Lent, R. W., Brown, S. D., \& Larkin, K. C. (1984). Relation of self-efficacy expectations to academic achievement and persistence. Journal of Counseling Psychology, 31(3), 356-362. doi:10.1037/0022-0167.31.3.356

Lipnevich, A. A., Maccann, C., Krumm, S., Burrus J., \& Roberts, R. D. (2011). Mathematics attitudes and mathematics outcomes of U.S. and Belarusian middle school students. Journal of Educational Psychology, 103(1), 105-118. doi:10.1037/a0021949 
Mayer, R. E. (1998). Cognitive, metacognitive, and motivational aspects of problem solving. Instructional Science, 26, 46-63. doi:10.1023/A:1003088013286

Neves, L. F. (2002). Um estudo sobre as relações entre a percepção e as expectativas de professores e dos alunos e o desempenho em Matemática (Dissertação de mestrado, Faculdade de Educação, Universidade Estadual de Campinas, SP, Brasil). Recuperada em http://www.bibliotecadigital.unicamp.br/document/?code=vtls000266066

Neves, L. F., \& Brito, M. R. F. (2001). Relações entre auto-eficácia matemática e desempenho em matemática de alunos do ensino fundamental [Resumo]. In Resumos de Comunicações Cientificas, XXXI Reunião Anual de Psicologia (p. 198). Rio de Janeiro, RJ: Fundação Carlos Chagas Filho de Amparo à Pesquisa do Estado do Rio de Janeiro.

Neves, S. P., \& Faria, L. (2007). Auto-eficácia académica e atribuições causais em Português e Matemática. Análise Psicológica, 4(25), 635-652.

Pajares, F. (1996). Self-efficacy beliefs and mathematical problem-solving of gifted students. Contemporary Educational Psychology, 21, 325-344.

Pajares, F. (2005). Self-efficacy during childhood and adolescence. Implications for teachers and parents. In F. Pajares \& T. Urdan (Eds.), Selfefficacy beliefs of adolescents (pp. 339-367). Greenwich, CT: Information Age.

Pajares, F., \& Graham, L. (1999). Self-efficacy, motivation constructs, and mathematics performance of entering middle school students. Contemporary Educational Psychology, 24, 124-139. doi:10.1006/ceps.1998.0991

Pajares, F., \& Miller, M. D. (1994). Role of selfefficacy and self-concept beliefs in mathematical problem solving: A path analysis. Journal of Educational Research, 86, 193-203. doi:10.1037/0022-0663.86.2.193

Pajares, F., \& Schunk, D. H. (2001). Self-beliefs and school success: Self-efficacy, self-concept, and school achievement. In R. Riding \& S. Rainer (Eds.), Perception (pp. 239-266). London: Ablex.

Rodrigues, L. C., \& Barrera, S. D. (2007). Auto-eficácia e desempenho escolar em alunos do Ensino Fundamental. Psicologia em Pesquisa, 1(2), 41-53.
Shavelson, R. J., Hubner, J. J., \& Stanton, G. C. (1976). Self-concept: Validation of construct interpretations. Review of Educational Research, 46(3), 407-441. doi:10.3102/00346543046003407

Shiomi, K. (1992). Association of attitude toward mathematics with self-efficacy, causal attribution, and personality traits. Perceptual and Motor Skills, 75, 563-567.

Skemp, R. R. (1971). The psychology of learning mathematics. Middlesex, UK: Penguin Books.

Smith, E. V., Jr., Wakely, M. B., de Kruif, R. E. L., \& Swartz, C. W. (2003, June). Optimizing rating scales for self-efficacy (and other) research. Educational and Psychological Measurement, 63(3), 369-391. doi:10.1177/0013164403063003002

Soares, A. B., \& Oliveira, M. B. (2011). Auto-eficácia, raciocínio verbal e desempenho escolar em estudantes. Psicologia: Teoria e Pesquisa, 27(1), 33-39. doi:10.1590/S010237722011000100005

Stevens, T., Olivarez, A., Lan, W. Y., \& TallentRunnels, M. K. (2004). Role of mathematics self-efficacy and motivation in mathematics performance across ethnicity. Journal of Educational Research, 97(4), 208-221. doi:10.3200/ JOER.97.4.208-222

Stirin, K., Ganzach, Y., Pazy, A., \& Eden, D. (2012). The effect of perceived advantage and disvantage on performance: The role of external efficacy. Applied Psychology: An International Review, 61(1), 81-96. doi:10.1111/j.14640597.2011.00457.x

Torisu, E. M., \& Ferreira, A. C. (2011). O papel das crenças de autoeficácia na motivação para aprender Matemática: Um estudo com alunos do Ensino Fundamental. Trabalho apresentado na XIII Conferência Interamericana de Educação Matemática, Recife, PE.

Williams, T., \& Williams, K. (2010) Self-efficacy and performance in mathematics: Reciprocal determinism in 33 nations. Journal of Educational Psychology, 102(2), 453-466. doi:10.1037/ a0017271

Wolters, C. A., \& Pintrich, P. R. (1998). Contextual differences in student motivation and selfregulated learning in mathematics, English, and social studies classrooms. Instructional Science, 26, 27-47. doi:10.1023/A:1003035929216 
Yailagh, M. S., Lloyd, J., \& Walsh, J. (2009). The causal relationships between attribution styles, mathematics self-efficacy beliefs, gender differences, goal setting, and math achievement of school children. Journal of Education \& Psychology, 3(2), 95-114.

Zimmerman, B. J., Bandura, A., \& MartinezPons, M. (1992). Self-motivation for academic attainment: The role of self-efficacy beliefs and personal goal setting. American Educational Research Journal, 29, 663-676. doi:10.3102/00028312029003663
Zimmerman, B. J., \& Campillo, M. (2003). Motivating self-regulated problem solvers. In J. E. Davidson \& R. J. Sternberg (Eds.), The psychology of problem solving (pp. 233-262). Cambridge, UK: Cambridge University Press.

Recebido: 28/09/2012

$1^{a}$ revisão: $12 / 05 / 2014$

Aceite final: 30/07/2014 